\title{
A Brief Discussion on the Technical Skill Difference Between Chinese Classical Dance and Western Dance
}

\author{
Na Yue \\ Xi'an University, Xi'an Shanxi, 710065, China
}

Keywords: Chinese classical dance, Western dance, Ballet, Technical skills.

\begin{abstract}
Chinese classical dance is a dancing art based on Chinese traditional culture inheritance and development, this kind of dance has beautiful dancing posture and unique technical skills, it has obvious difference with western dance on movement, movement rules, power generation and performance, which formed an unique dance system. This paper starts from the technical skills of Chinese classic dance to make a difference analysis on the technical difference between Chinese classical dance and western dance.
\end{abstract}

\section{Technical skills of Chinese classical dance}

Throughout the development history of Chinese classical dance, developed from initial "wholesale dramatization" to the inclusive development with traditional drama, martial art and ballet, which promoted Chinese classical dance formed an independent art system, it found its own development path. The technical skills of Chinese classical dance are mainly showed on the three aspects of "jump, spin and turn over", these three skills constructed the technical skill system of Chinese classical dance, though they are with different embodiment, they abide by same art base and common aesthetic principle.

\section{Technical skill of jump}

Jump is the skill base of Chinese classical dance for creating elegant artistic conception, showing explosive power of flash and also strengthening flying height, giving people the feeling that light as a swallow. Chinese classical dance inherited the technical skills of traditional dramatic martial, enriched the technical skill system, jump of pelvic limbs and the pitching of body formed a very unique modeling feature. Classify from modeling features jump skill can be divided as basic jump, dancing jump, their technical skills are as followings:

1.Basic jump. The basic jump of Chinese classical dance imitated part of basic jump teaching experience of ballet, without form change of body, pay attention to ankle's power and speed, including double rise and fall jump, single rise double fall jump, middle jump double rise and fall, double rise and fall trestle jump and so on. Small jump focus on feet's power and flexibility, it is base for middle and big jump. Middle jump focuses on knee and leg muscle power, big jump focuses on the embodiment of body language in the air, requiring a certain jumping range.

2.Dancing jump. Dancing jump changes according to different national dancing postures, combining body to show jumping, strengthening pelvic limbs' match, fully expresses the Yin and Yang relationship in Chinese traditional culture. Dancing jump strengthens to find balance in imbalance, realize perfect matching of jump and body in the flash of power explosion. Upper body mainly includes technical skills such as twisting, leaning, bowing, face upward, open, close and son on, in the dancing jump process, dancers need to deal with master-slave relation between dancing jump and upper body, this has an obvious difference with the straight jump of western ballet. 


\section{Technical skill of spinning}

Spinning is a normal skill in dancing performance, spinning skills in classical dance has very obvious features this kind of skill is not isolated functional training, it includes distinctive dance language. Generally speaking, we can divide spinning skill of classical dance into following three forms:

1.Straight spinning. It is also called as basic spinning, is the basic link of dance spinning skill, can be divided as on site straight spinning and advancing straight spinning specifically, the former means body keeps straight, body spin on site without any elements, squatting is the applying force for this spinning, because dancers' upper body is relative solid, they are easy to find barycenter; the latter means flat spinning and upper assisting leg spinning, flat spinning is formed barycenter exchange on the basis of two feet, upper assisting leg is formed by setting single foot as moving barycenter.

2.Dancing spinning. This spinning is based on straight spinning, using arms, legs and body to form different spinning. According to range, it can be divided into big dancing spinning and small spinning, this kind of spinning is with strong mobility, during dancing performance it need a certain space, therefore its dance language is improved obviously. During spinning, dancing spinning no need to play dancing posture well, but to show all kinds of dancing postures during spinning, this has a high requirement on the dancing posture accuracy, competency, elegance and flency.

3.Composite spinning. It is a complex form of single spinning, it should be pointed out that composite spinning is not just an overlay of several single spinning skills, but to link between two or more different spinning skills through action and dancing postures. Classical dance performance requires a satisfactory aesthetic state, this is more highlighted in spinning skill, while composite spinning is an organic link for different sinning forms, it requires dancers should connect next spinning action during the three-quarter of the first spinning to make two spinning skills to form one composite skill naturally, for dancers, grasping this spinning skill need a long progress but cannot realize in a short time, it has high requirement on dances' body copperation, psychological quality and other aspects.

\section{Technical skills of turning over}

Turing over is a special technical skill for Chinese classical dance, it absorbed action tips of Chinese drama and martial art. Turning over requires dancers to turn over with vertical axis to form circling motion under the leaning state. The skill center of turning over is on pelvis, during turning over dancers should make arm action through the circle path. In Chinese classical dance, turning over has extremely art expressive force, combining it with body can fully express dancers' skill features of twisting, leaning, circle and flexural. Turing over is with various skills, which can produce different art effect and express dancers' emotion change, it mainly include following skills:

1.Turning over with feet as barycenter. This kind of turning over specifically mean turning over without squat, dynamic foot forms step beside the power foot, two feet are barycenter. Short turning over is an expression way of step turning over, its skills are basically same with steps turning over, the only difference is that steps with bigger range, range of both knees is deeper.

2.Turning over with one foot as barycenter. Dot step turning over and knee lift turning over are main expression of single foot turning over, the former set power leg as axis, and touch floor with power leg, continue to top hip, throw head to form continuous turn over, it is basic link of single foot turning over; the latter need to be completed on the basis of dot step turning over, this skill take power leg as support to complete turning over.

3.Movement barycenter turning over. Bunch turning over is expression of movement barycenter turning over, means arms through four turning over process and movement barycenter turning over. This skill needs two feet continue move to complete barycenter exchange and keep move forward, pick waist and bare-chest are key points of this skill.

4.Dancing posture turning over. Creeping turning over is main expression of dancing posture turning over, this is a kind of turning over skill with national aesthetic features, relatively slow on speed, skill is difficult, with strong feminine features and strong expression. 


\section{Technical skill difference between Chinese classical dance and western dance}

Chinese classical dance not only inherited technical skills of Chinese traditional drama and martial art, but also referred teaching system of western classical ballet art to form its own unique style and various movement dance art. Influenced by different Chinese and western aesthetic value, Chinese classical dance and western dance not only different on expression style but also have obvious difference on technical skills, specifically expressed in following aspects:

\section{The difference of body shape and technical skills}

Chinese classical dance has strong art expressive force, influenced by "virtuality and reality combination" aesthetic ideology, Chinese classical dance mainly expresses "round", that is dancers' body shape and movements should express "round" track. As followings: "flat round" requires movement tracks of arms should be like millstone; "figure eight round" requires arms or shoulders to make round movement with adverse direction within same period, guarantee movement crisscross and overlap to be like vehicle wheel; "vertical round" requires arms and legs form circle movement. Above "three round" are basic technical movement in Chinese traditional dance can shape most elegant dancing posture.

Western classical ballet strengthens shape to be tall and straight up, technically requires body to be "straight up", help people feel tall and straight. In dancing process, dancers abide by dance with "straight line" track, and based on this to extend dance movement. From whole ballet to see that dance skills focus on "barycenter straight", typical dancing posture Arabesque expressed application of "straight" skill.

\section{Difference of movement skills}

Power generates on twist in Chinese classical dance, start from twist to drive dynamic elements in the whole body, this also refers technical thoughts of Chinese traditional drama and martial art, which can endow dance with artistic appeal. In Chinese classical dance training, dancers usually take twist as key training part. But in western classical ballet movements, dancers pay attention to arms and legs movement, especially for "toes", it is one of the most obvious movement parts, generate power from toe, pay much attention on the technical movement of toes to express linear extension of aesthetic feeling.

\section{Technical skill difference of lower limbs}

Lower limb movements of Chinese classical dance is divers, it requires to express mobility dance movement through feet movements. Feet movements mainly include hooking foot, based on the order that heel touching floor, generally speaking, walking distance is relatedly small. Foot technical skills normally abide by the principle of body movement guide foot movement, adjust body barycenter with actions, thus to express diversity. It follows that lower limbs skills in Chinese classical dance strengthens beauty of dance, this beauty shows Chinese classical dance's elegant, connotation and the flexibility and light of dance art.

Lower limbs skills of western classical ballet are also diverse, from feet movement, it has different skills of "open, stretch tight, straight, erect", meanwhile, these technical movements have fix movement pattern. Sole and toe are parts that contact most with floor, and sole and toe are with huge movement range, relative height is rather high. Especially for "toe" movement, it strengthens light, fast skill movement, this is also a bright spot of ballet pace. Ballet has always chase for expression with "vertical center of gravity" to show light beauty of dance.

\section{Difference of performance features}

Chinese classical dance and western dance has obvious difference on performance, they express different arts beauty, the following will set classical dance "Toka" and "Swan Lake" as example to discuss differences between two kinds of dances. 
"Toka" strengthens singing and dancing joyously on performance, dancers wear green long sleeve dance clothes, use same foot to create rhythm, accompany with simple background to create image of furious spring. Spontaneous expression and abstraction are key points of this classical work, only few dancers can express a group of people go on a spring outing. From the perspective of aesthetic sense, this work pay attention to virtual and real realm, besides steps, this dance also has some mobile pace, whole pause formed instantaneous flow, and formed visual contract through contrast of pause and flow. If there is a small part in this work that dancers twist waist to left direction, throw out sleeves, long sleeves shows arrow image, in the split second that to the left side, turn right, when sleeves fall down, dancers body suddenly turn left, in this process, dancers like floating clouds and flowing water, at this time, all movements will be completed in the lyric "I am always right here with you". During the performance, dancer team always change in a symmetrical form, this is also the most outstanding point of this dance, this feature is matched with Chinese traditional moderation.

"Swan Lake" is representative work of western ballet, as long as mentioned swan lake, people will associate with ballet, this work is crystal of many masters, such as Tchaikovsky's music, Ivanov's impressionist creation and so on, it is famous to the world with its idyllic dance. In performance form, this dance has both solo dance, pas de deux and group dance, classical ballet is fully displayed here. There is a typical group dance in Charter II of "Swan Lake", which is with strong impact, 24 dances play swan in an orderly arrangement, dancers make full use of the stage space, foiled the dancing atmosphere, and further strengthen impact to express objective substantive in western culture, this kind of performance has obvious difference with Chinese classical dance.

\section{Conclusion}

All in all, during development Chinese classical dance formed unique technical skill system, it not only inherited Chinese traditional drama and martial technical movement essence, but also referred to the construction experience of western classical ballet, strengthened the art expressive force of Chinese classical dance, expressed diverse development style and features. Chinese classical dance is typical representative of Chinese classical dance culture, it focused on broadcasting oriental culture through technical skills, expressed deep human thought, therefore, Chinese classical dance should always insist national cultural features, clearly realized the difference between Chinese classical dance and western dance to carry forward Chinese classical dance.

\section{References}

[1] Can Mengxin. Discuss on the Organic Combination of Chinese Classical dance and Modern dance, Canhua,2016(11):102-103.

[2] Yang Jingjing. Exploration and Thinking on Chinese Classical Training, Sichuan Normal University, 2014.

[3] Yu Ping. Chinese Classical Dance in the View of Discipline Construction, Journal of Beijing Dance College, 2016(4):69-70.

[4] Duan Ziyu. Conflict and Integration of Chinese and Western Culture-Set Physical Dance as Example, Wuhan Physical Training College, 2014(5):56-57.

[5] Zhang Jinjiang. Understand Regional Aesthetic Orientation from Chinese and Western Dance Style Difference, The Sound of Huanghe, 2016(5):65-66. 